\title{
Hallux valgus and plantar pressure loading: the Framingham foot study
}

\author{
Andrew M Galica ${ }^{1 \dagger}$, Thomas J Hagedorn ${ }^{1 \dagger}$, Alyssa B Dufour ${ }^{1,2,3}$, Jody L Riskowski ${ }^{4}$, Howard J Hillstrom ${ }^{5}$, \\ Virginia A Casey ${ }^{1}$ and Marian T Hannan ${ }^{1,2,3^{*}}$
}

\begin{abstract}
Background: Hallux valgus (HV), a common structural foot deformity, can cause foot pain and lead to limited mobility. The purpose of this study was to evaluate differences in plantar pressure and force during gait by HV status in a large population-based cohort of men and women.
\end{abstract}

Methods: A trained examiner performed a validated physical examination on participants' feet and recorded the presence of hallux valgus and other specific foot disorders. Each foot was classified into one of four mutually exclusive groups based on the foot examination. Foot groups were: (i) HV only, (ii) HV and at least one additional foot disorder (FD), (iii) no HV but at least one other FD, and (iv) neither HV nor FD (referent). Biomechanical data for both feet were collected using Tekscan Matscan. Foot posture during quiet standing, using modified arch index (MAI), and foot function during gait, using center of pressure excursion index (CPEI), were calculated per foot. Further, walking scans were masked into eight sub-regions using Novel Automask, and peak pressure and maximum force exerted in each region were calculated.

Results: There were 3205 participants, contributing 6393 feet with complete foot exam data and valid biomechanical measurements. Participants with HV had lower hallucal loading and higher forces at lesser toes as well as higher MAI and lower CPEI values compared to the referent. Participants with HV and other FDs were also noted to have aberrant rearfoot forces and pressures.

Conclusions: These results suggest that HV alters foot loading patterns and pressure profiles. Future work should investigate how these changes affect the risk of other foot and lower extremity ailments.

\section{Background}

Hallux valgus (HV), a structural foot deformity often resulting in a reactive soft tissue bunion, can cause foot pain and limited mobility [1]. Women are twice as likely to have this condition [2,3] and older adults have a higher prevalence of HV ( $23 \%$ aged $18-25$ years versus $35.7 \%$ over age 65 years) [4]. Footwear has also been implicated in the development of HV; especially shoes with higher heels or improper fit [5].

The degree to which foot anatomy or biomechanics influence HV is poorly understood. In a 2012 systematic review and meta analysis, Nix et al. reported that the first intermetatarsal angle and first metatarsal protrusions

\footnotetext{
* Correspondence: hannan@hsl.harvard.edu

${ }^{\dagger}$ Equal contributors

'Institute for Aging Research at Hebrew Senior Life, 1200 Centre Street, Boston, MA, USA

${ }^{2}$ Beth Israel Deaconess Medical Center, Boston, MA, USA

Full list of author information is available at the end of the article
}

distance were significantly associated with hallux valgus, but also noted that a number of radiographic factors were not significantly associated with hallux valgus [6]. Arch height is noted as an area of interest in clinical models of hallux valgus [7], which often cite low arches as a contributing factor. However, past research has yielded inconsistent results [6]. While Nguyen et al. found a significant association between a clinical assessment of pes planus and hallux valgus in men [2], Kilmartin et al. reported no relation between arch height and hallux valgus when using an arch index [8]. Similarly, studies are inconsistent regarding whether a curved joint head was [9] or was not [10] associated with HV.

Studies that report on plantar pressure distributions for individuals with and without HV are also inconclusive. For example, loading at the hallux may be reduced [11-13], increased [14], or unaffected [15] by the presence of HV. These studies were limited by small sample 
sizes [14-16] and narrow age ranges [15,17], as well as exclusions of severe clinical cases [14], men [18], and feet with multiple foot disorders [18].

Prior studies are limited by conflicting results, small sample sizes, and consideration of hallux valgus in isolation of concurrent foot disorders. Addressing these limitations can impact clinical decision making and evaluation of treatment strategies. The purpose of this study is to describe plantar pressures and forces in a large epidemiologic, population-based study of older adults and to investigate whether these measures differ between those with and without $\mathrm{HV}$ and other foot deformities. We hypothesized that the presence of $\mathrm{HV}$ is associated with decreased loading under the hallux and resultant offloading under the forefoot.

\section{Methods}

\section{Study population}

Study participants were members of the Framingham Foot Study [18], a population-based study comprised of three cohorts: Framingham Original Cohort, Framingham Offspring Cohort and a community sample drawn from the town of Framingham, MA, USA [18]. Briefly, the Framingham Original Cohort was derived in 1948 from a two-thirds population-based sample of the town of Framingham, MA, USA, while the Framingham Offspring cohort was composed of a sample of the adult children and spouses of the Original Cohort. Members of these cohorts between 2002-2008 were included in the Framingham Foot study along with a newly recruited community sample. The Framingham community sample was recruited via census-based, random digit-dial of ambulatory residents who were age 50 or older. The Framingham Foot Study was approved by the Institutional Review Boards at Hebrew SeniorLife and Boston University. All study participants provided written, informed consent prior to enrolment. Between 2002 and 2008, Foot Study participants received a physical and biomechanical assessment of their feet. For this analysis, only Framingham Foot Study participants with complete foot biomechanical data and foot disorder data were included.

\section{Hallux valgus and other foot disorders}

A podiatric-trained examiner performed a validated physical examination on participants' feet and recorded the presence of specific foot disorders including hallux valgus, hallux rigidus, claw toes, hammers toes, and overlapping toes [2]. The validity of the foot exam was previously evaluated in a sample of elderly residents by comparing podiatry clinic findings to the results from the trained study examiners. There was excellent agreement for $\mathrm{HV}$ as well as other foot disorders that were included in the foot examination. A comparison of multiple examiners yielded kappa values $>0.85$ (all $p<$ 0.01 ), and all domains demonstrated excellent interobserver and intraobserver reliability $[19,20]$. Presence of HV (yes/ no) was defined as a $15^{\circ}$ or greater abduction of the hallux with respect to the first metatarsal. While the participant was standing, the examiner compared the angle of the hallux to an illustration of a $15^{\circ}$ angle printed on a laminated page, and recorded hallux valgus as present if the angle was larger than the illustration. Hallux valgus, hammer toes, claw toes, and overlapping toes were assessed during weight-bearing stance. Hallux rigidus was measured while the participant was non-weight bearing and was considered present if the hallux was frozen or rigid during attempted passive movement by the examiner. All foot disorders (FD) were recorded as present or absent.

Each foot was classified into one of four mutually exclusive groups based on the physical examination. The foot groups were defined as: (i) hallux valgus only (HV-only), (ii) $\mathrm{HV}$ and at least one additional foot disorder (HV + FD), (iii) no HV but at least one other foot disorder (no HV-FD only), and (iv) no-HV and no-FD (referent group).

Age, sex and weight were also recorded at the time of examination. Weight was measured to the nearest half pound using a standardized balance beam scale and converted to Newtons.

\section{Plantar pressure data collection}

Plantar pressure data were collected using a Tekscan Matscan (Tekscan Inc., Boston MA) pressure mat with a capture rate of $40 \mathrm{~Hz}$, which was sufficient for the type of data collected and has moderate to good reliability [21]. A scan of each participant in quiet, bipedal stance was collected. Additionally, participants were instructed to walk at a self-selected pace across the mat. A single pressure scan of each foot was recorded using the twostep method [22], which entails the participants stepping on the pressure map on the second step. The two-step method has been shown to be as reliable as data collection using the mid-gait approach [22]. The time constraints associated with a large epidemiological study allowed recording of a single walking trial.

\section{Plantar pressure analysis and outcome measures}

Walking scans were masked into eight sub-regions using Novel Automask (Novel GmbH, Munich, Germany), and the peak pressure and maximum force exerted in each region were calculated. The eight regions were: (i) hallux, (ii) lesser toes, (iii) lateral forefoot, (iv) medial forefoot, (v) lateral midfoot, (vi) medial midfoot, (vii) lateral rearfoot, and (viii) medial rearfoot (Figure 1).

In addition to regional pressures and force measurements, the center of pressure excursion index (CPEI) [23], a measure of foot function during gait [23], and the 


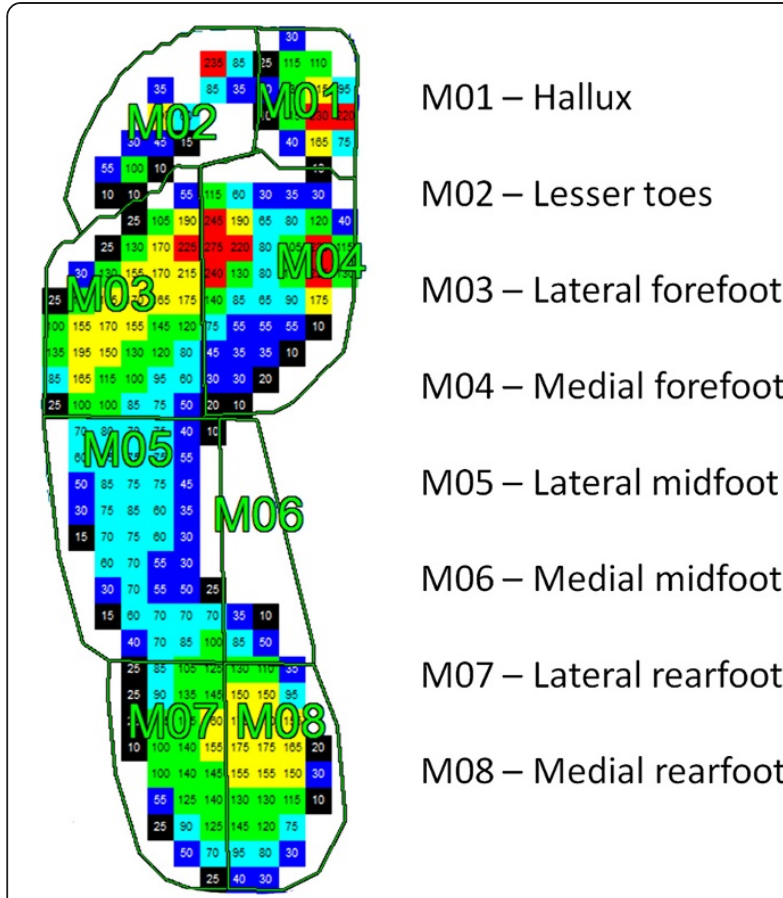

Figure 1 The eight region foot mask used to analyze Matscan plantar pressure scans in the Framingham Foot Study, 2002-2008.

modified arch index (MAI), a measure of foot structure [24], were calculated for each foot. CPEI is related to clinical foot type and was defined as the excursion of the center of pressure from a constructed line connecting the first and last points of a center of pressure curve measured in the distal tertile of the foot and normalized by the foot's width [23]. Lower CPEI values indicate a more pronated foot during gait, whereas higher CPEI values indicate more supination (Figure 2). MAI, calculated from the static weight-bearing stance scan was calculated by dividing each foot, not including the toes, lengthwise into three equal segments. The pressure under the middle third of the foot was divided by the pressure under the entire foot to yield the MAI [24]. Previous work has shown that MAI was inversely associated with navicular height, with higher MAI values indicating a lower arch [25].

\section{Statistical analysis}

Means and standard deviations, or frequencies, where appropriate, were calculated for the overall population, and separately by foot group. A per-foot analysis using linear regression was used to determine the association between each biomechanical measure and foot group, both crude and adjusted (age, sex, and weight [Newtons]). Generalized Estimating Equations (GEE) were used to account for the correlation between right and left foot of the same person. The dependent

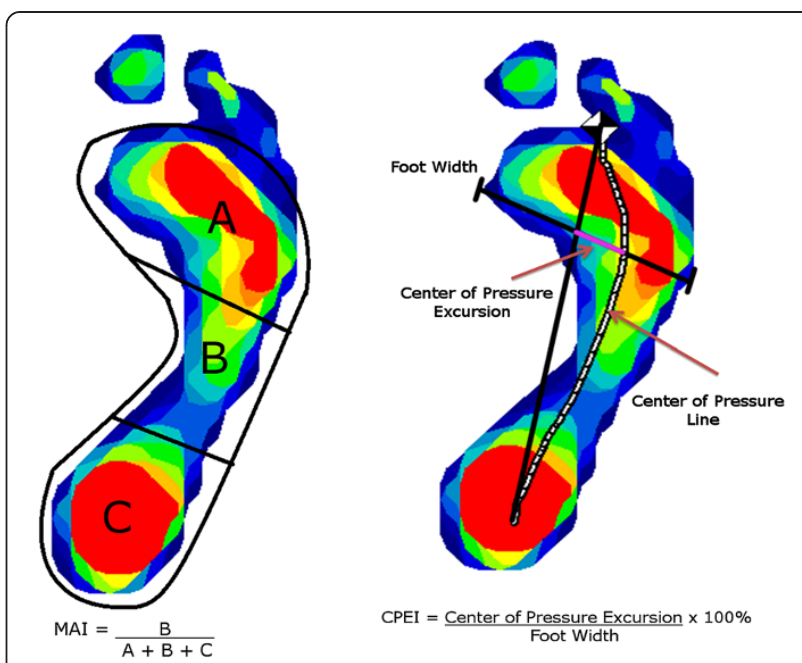

Figure 2 Calculation of the center of pressure excursion index (CPI) and the modified arch index (MAI) in the Framingham Foot Study, 2002-2008. Used with permission by Arthritis Care \& Research; John Wiley \& Sons, Inc.

variables were peak pressure and maximum force in each of the foot regions, along with CPEI and MAI. Results were considered statistically significant at $p<0.05$. $P$-values from the linear regression models were adjusted for the 8 comparisons made within the mask in models of peak pressure and maximum force using a Bonferroni correction for multiple comparison testing. $P$-values from models of CPEI and MAI were not adjusted. The results presented account for the correction for multiple testing. All statistical analyses were conducted using the SAS statistical analysis package, version 9.3 (SAS Institute, Cary, NC).

\section{Results}

Population

There were 3205 participants, contributing 6393 feet with complete foot exam data and valid biomechanical measurements (Figure 3). The average age was 66 years and $56 \%$ of the sample was female (Table 1).

\section{Maximum forces}

Statistically significant differences in maximum force under the hallux were seen in both HV groups (i.e., HVOnly, HV + FD), but not in the No-HV + FD after adjustment for age, sex, and weight (Table 2). In addition to reduced loading under the hallux, the HV-only group had increased loading under the lesser toes in both crude and adjusted models. Though crude associations suggested decreased loading in additional regions, the HV-only group was not significantly different from the referent group at the other masked regions in adjusted models. By comparison, the HV + FD group had significantly reduced loading under both the lateral forefoot 


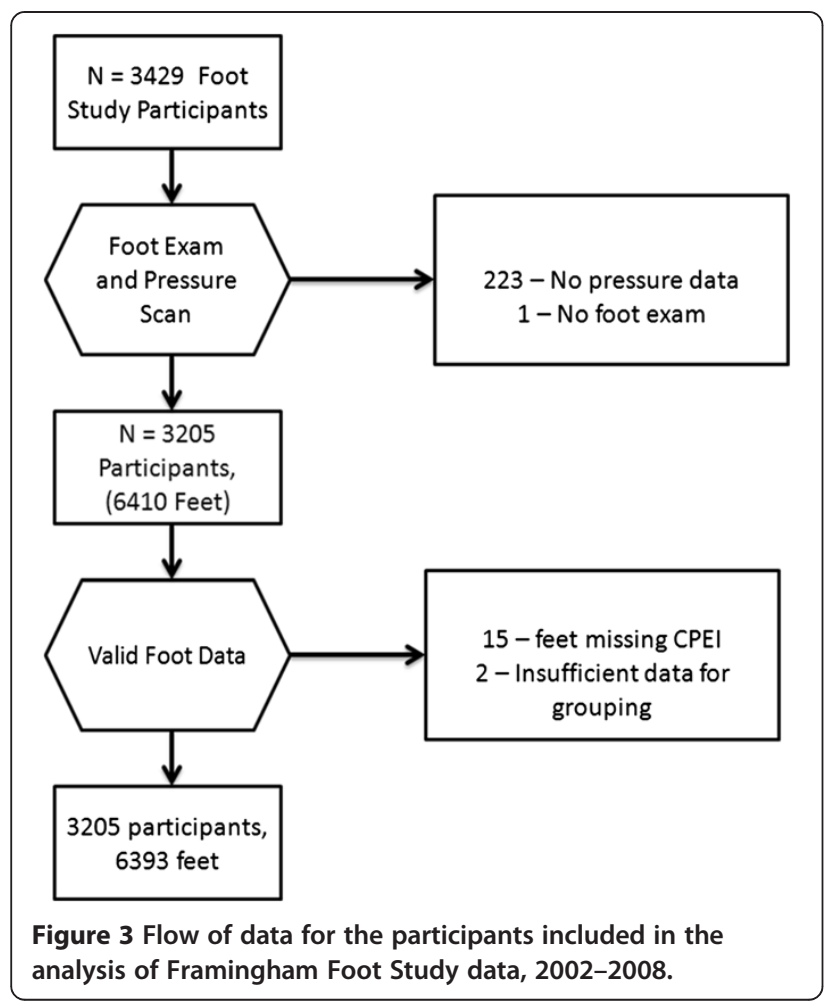

and lateral rearfoot in addition to the aforementioned reduction under the hallux in both crude and adjusted models. After adjustment, the HV + FD group did not differ significantly from the referent in any other regions. The No-HV + FD group did not have any statistically significant differences in force in the adjusted models relative to the referent group, though there were significant crude associations. No significant differences were seen for any of the foot groups at the lateral or medial midfoot.

\section{Peak pressures}

There were no significant differences in peak pressure observed in the HV-only group in crude or adjusted models, but several differences were noted in the $\mathrm{HV}+$ FD group. In this group, in both crude and adjusted models, peak pressure was significantly reduced under the hallux, increased under the lesser toes, and reduced in both the medial and lateral rearfoot. In the No-HV + FD group, a similar increase in pressure was observed under the lesser toes, but no changes were observed in any other masked region.

\section{Center of pressure excursion index (CPEI) and modified arch index (MAI)}

CPEI was significantly reduced in both the HV-only and HV + FD groups, but was not statistically significantly different in the No-HV + FD group. MAI, by comparison, was not significantly different in the HV-only group, but it was higher than the referent in the HV + FD and the No-HV + FD groups. These associations were maintained in both crude and adjusted models.

\section{Discussion}

The purpose of this study was to evaluate the differences in plantar pressure and force during gait by hallux valgus (HV) status in a large population-based cohort of men and women. Our results show that loading in the hallux region was lower in participants with HV-only and in those with $\mathrm{HV}$ and other foot disorders, compared to those who had neither. All groups (HV-only, HV + FD, and FD-only) had greater loading or pressure in the lesser toe regions when compared to the referent. The HV + FD group also had lower lateral rearfoot maximum force and lateral and medial peak pressures relative to the referent. Furthermore, feet in the HV + FD group were more likely to display a lower center of pressure excursion index (CPEI) values, higher modified arch index (MAI) values, and reduced lateral rearfoot force and lowered rearfoot peak pressures compared to the referent group. These results suggest that feet with HV have altered loading patterns and pressure profiles that may put them at greater risk of other foot and lower extremity ailments.

\section{Hallux and lesser toes}

Our study showed reduced force under the hallux in both HV groups (i.e., HV-only and HV + FD), but not in the No-HV + FD group. Pressure was also reduced under the hallux in the HV + FD group. While reduced pressure under the hallux in those with hallux valgus

Table 1 Characteristics of the Framingham foot study population, 2002-2008*

\begin{tabular}{|c|c|c|c|c|c|}
\hline & Population & $\begin{array}{l}\text { No Hallux Valgus/No } \\
\text { foot disorder (referent) }\end{array}$ & Hallux Valgus only & $\begin{array}{l}\text { Hallux Valgus + } \\
\text { foot disorder }\end{array}$ & Foot disorder only \\
\hline $\mathrm{N}$ feet & 6393 & 3707 & 1123 & 641 & 922 \\
\hline Age, years & $66.2 \pm 10.5$ & $64.3 \pm 9.7$ & $65.2 \pm 10.0$ & $73.4 \pm 10.9$ & $69.9 \pm 10.9$ \\
\hline Female $(n, \%)$ & 1799 (56.1\%) & 1849 (49.9\%) & $832(74.1 \%)$ & $462(72.1 \%)$ & $448(48.6 \%)$ \\
\hline Weight, pounds & $174.1 \pm 39.5$ & $178.4 \pm 39.5$ & $164.4 \pm 36.0$ & $161.7 \pm 38.9$ & $177.3 \pm 39.9$ \\
\hline $\mathrm{BMI}, \mathrm{kg} / \mathrm{m}^{2}$ & $28.4 \pm 5.5$ & $28.8 \pm 5.5$ & $27.7 \pm 5.2$ & $27.5 \pm 5.4$ & $28.5 \pm 5.3$ \\
\hline
\end{tabular}

*Data reported as mean \pm standard deviation, unless otherwise noted. 
Table 2 Distributions of foot biomechanical variables in each of the four foot groups, Framingham foot study, 2002-2008

\begin{tabular}{|c|c|c|c|c|}
\hline Variable & $\begin{array}{l}\text { No Hallux Valgus / No } \\
\text { foot disorder (referent) }\end{array}$ & Hallux Valgus only & $\begin{array}{l}\text { Hallux Valgus + } \\
\text { foot disorder }\end{array}$ & Foot disorder only \\
\hline \multicolumn{5}{|l|}{ Maximum Force $(\mathrm{N})$} \\
\hline Hallux & $78.4(39.70)$ & $64.9(41.72)^{\dagger *}$ & $47.7(33.47)^{\dagger *}$ & $70.4(42.28)^{\dagger}$ \\
\hline Lesser Toes & $41.1(27.12)$ & $45.3(38.04)^{*}$ & $40.7(25.91)^{\dagger}$ & $38.1(27.83)$ \\
\hline Lateral Forefoot & $249.7(102.50)$ & $225.7(104.30)^{\dagger}$ & $206.2(84.81)^{\dagger *}$ & $229.8(110.81)^{\dagger}$ \\
\hline Medial Forefoot & $132.9(72.03)$ & $123.1(63.02)^{\dagger}$ & $116.4(62.89)^{\dagger}$ & $130.3(76.12)$ \\
\hline Lateral Midfoot & $116.5(94.35)$ & $108.3(93.37)$ & $109.5(76.57)$ & $124.4(112.22)$ \\
\hline Medial Midfoot & $18.8(24.62)$ & $21.0(32.87)$ & $20.0(24.17)$ & $21.6(27.84)$ \\
\hline Lateral Rearfoot & $175.6(63.30)$ & $163.9(59.92)^{\dagger}$ & $151.9(51.41)^{\dagger_{*}}$ & $167.1(70.77)^{\dagger}$ \\
\hline Medial Rearfoot & $196.5(68.11)$ & $181.8(65.06)^{\dagger}$ & $172.6(56.38)^{\dagger}$ & $191.9(73.32)$ \\
\hline \multicolumn{5}{|l|}{ Peak Pressure (kPa) } \\
\hline Hallux & $218.6(102.01)$ & $212.0(106.44)^{\dagger}$ & $171.3(99.38)^{*}$ & $206.0(106.66)^{\dagger}$ \\
\hline Lesser Toes & $138.8(77.36)$ & $144.5(87.82)^{\dagger}$ & $148.3(71.93)^{*}$ & $152.2(90.16)^{\dagger *}$ \\
\hline Lateral Forefoot & $251.3(88.12)$ & $240.3(89.11)^{\dagger}$ & $231.5(71.15)^{\dagger}$ & $249.6(88.87)$ \\
\hline Medial Forefoot & $212.8(101.25)$ & 206.5 (99.94) & $207.2(86.07)$ & $222.0(104.89)^{\dagger}$ \\
\hline Lateral Midfoot & $108.8(64.45)$ & $106.3(74.1)$ & $107.8(61.02)$ & $113.1(72.42)$ \\
\hline Medial Midfoot & $74.4(53.20)$ & $73.5(65.18)$ & $68.1(39.27)$ & $73.4(55.17)$ \\
\hline Lateral Rearfoot & $216.8(80.05)$ & $208.2(80.42)^{\dagger}$ & $200.4(62.83)^{*}$ & $213.1(83.86)$ \\
\hline Medial Rearfoot & $230.0(85.98)$ & $222.3(84.72)^{\dagger}$ & $215.3(67.21)^{*}$ & $233.1(92.31)$ \\
\hline Center of Pressure Excursion Index & $14.7(8.07)$ & $13.0(8.00)^{\dagger *}$ & $12.5(7.74)^{\dagger *}$ & $14.5(8.00)$ \\
\hline Modified Arch Index & $0.102(0.0809)$ & $0.098(0.079)^{\dagger}$ & $0.112(0.095)^{*}$ & $0.116(0.099)^{\dagger *}$ \\
\hline
\end{tabular}

Means (standard deviations).

$\mathrm{CPEI}=$ center of pressure excursion index.

$t=$ significantly different from referent group in crude model at $p<0.05$ with Bonferroni adjustment.

* = significantly different from referent group in adjusted model (age, weight, sex) at $p<0.05$ with Bonferroni adjustment.

has been seen in previous studies [13,17], this result has not been reported consistently [26]. Past studies have also observed no significant difference in loading of the hallux [15], as well as an increase in pressure under the hallux [14]. The conflicting findings noted by MartinezNova et al. [14] could be due to the inclusion of only mild cases of hallux valgus and a comparatively smaller, younger cohort (mean age 54.7 years). Biomechanical studies have suggested a number of mechanisms to explain the reduced loading in the hallux region in feet with HV, including first ray hypermobility. In this model the first metatarsal gives way, resulting in an offloading of the hallux onto other aspects of the foot such as the second metatarsal $[9,16,17,27]$. The offloading of the hallux may be due to the reduced ability of the hallux to bear load [27], or an adaptation to pain. Correspondingly, we found reduced loading at the hallux region with $\mathrm{HV}$, while maximum force in the lesser toe area was increased in the HV-only group. As this study is cross-sectional is it unclear if the differences in halluca 1 loading associated with $\mathrm{HV}$ are a result of HV or causative of it.

We also noted higher peak pressure under the lesser toes in those feet with other structural foot disorders (i.e., HV + FD and No-HV + FD groups). As noted above, the offloading of the hallux may be due to its reduced ability to bear load [27], or as an adaptation to pain. In the HV + FD and No-HV + FD groups, the lack of significant changes in force at the lesser toes points to a different explanation. As the other foot disorders considered in this study are primarily structural disorders of the toes, it is possible that the contact area under the toes is reduced in these two groups as a result of other foot disorders, which can explain increases in pressure despite the lack of significant changes in force in those with the other structural foot disorders.

\section{Forefoot}

Prior studies of surgical patients have also reported that $\mathrm{HV}$ is associated with lower loading at the $1^{\text {st }}$ metatarsophalangeal joint (MTPJ) [11,12]. This reduced loading has been theorized to result from an elevation of the $1^{\text {st }}$ MPJ during gait, which limits the load this area is able to accept in those with HV $[16,17,28]$. Studies in nonclinical groups by comparison have noted an increased load at the $1^{\text {st }}$ MTPJ $[15,16,26]$. In this analysis, there were no differences with adjusted models in the medial 
forefoot in any of the foot disorder groups. As the masking used in this study did not differentiate between the individual metatarsals, it possible that the offloading of the first metatarsal head was offset by increased force on the $2^{\text {nd }} M P J$. Nonetheless, no significant differences were seen in the medial forefoot, even in feet with HV. While no changes were observed in the medial forefoot, the HV + FD group had significantly reduced loading in the lateral half of the forefoot. Lower force in the lateral forefoot has not been previously attributed to HV. One explanation for the lower loading in the lateral forefoot could be a more pronated foot during gait, which is typically thought to be associated with HV. This hypothesis was supported by the significantly lower CPEI value observed in the groups with HV.

\section{Rearfoot}

Of the studies that have reported on the midfoot and rearfoot regions, there was no subdivision of these areas into a medial and lateral section [16,29], and only one study [29] showed a difference in either the midfoot or rearfoot pressures between feet with and without HV. Nyska et al. noted that feet prior to undergoing corrective surgery for $\mathrm{HV}$ displayed lower rearfoot pressure relative to those without the foot disorder [29]. In our study, a lower maximum force in the lateral rearfoot and lower rearfoot peak pressure were observed in the $\mathrm{HV}+$ FD group. A similar association of a medial to lateral loading pattern in people with rheumatoid arthritis suggests the rearfoot and forefoot may be coupled and the rearfoot may play a role in forefoot complications [30]. The medial to lateral loading pattern arises from a valgus rearfoot alignment and leads to excessive stress at the subtalar joint and forefoot region [31]. Although rearfoot alignment was not evaluated in this current study, the lower values of the CPEI noted in the groups with $\mathrm{HV}$ suggest that they displayed a valgus rearfoot alignment [23]. In short, these results suggest that the rearfoot may be an important factor in the etiology and treatment of HV. This novel aspect of our findings would need further investigation of these patterns of results in future studies.

\section{Center of pressure excursion index (CPEI) and modified arch index (MAI)}

Biomechanical modelling of $\mathrm{HV}$ has reported associations of pes planus foot type with the etiology of $\mathrm{HV}$ [28]. However, clinical and biomechanical studies of $\mathrm{HV}$ have not yielded consistent results to implicate a particular foot type with $\mathrm{HV}[7,32]$. In this current study, a smaller CPEI was observed in both HV-only and $\mathrm{HV}+$ FD groups. This indicates that participants with $\mathrm{HV}$ have a more pronated foot during gait relative to the referent group and may be more likely to have a pes planus foot type, which has been associated with a lower CPEI [23]. This observation is further supported in the $\mathrm{HV}+$ FD group by higher MAI values, indicating a lower arch in this group relative to the referent. These results offer support that $\mathrm{HV}$ is associated with differences in foot structure and function. Further research is needed to expand these novel findings.

\section{Strengths and limitations}

While this study provides important insights into the foot biomechanics associated with hallux valgus, there are several limitations. As the study design was crosssectional, causal relations cannot be inferred. Nonetheless, associations between foot groups and loading offer an epidemiological view of common loading profiles in those with HV and foot disorders. Only a single walking trial for each foot was recorded, which may have increased measurement error. However, random error would only serve to obscure weaker relations in these data rather than create false positive associations [33]. Moreover, the large study sample greatly mitigates this issue, as it is more than sufficiently powered even with a single scan [34].

As a common mask was used to define foot regions for all pressure scans, it is possible that foot regions may not have correlated exactly with the anatomical location of the corresponding metatarsals in some cases. To more accurately define foot regions, it may be useful in future studies to align anatomical foot structure from spiral X-ray tomography with plantar pressure data as described by Hastings et al. [35].

Although studies have previously addressed the topic of plantar loading in $\mathrm{HV}$, our study was unique in that it was population-based and included both men and women and individuals with additional foot disorders. Recent studies of plantar loading have been limited by relatively small samples ( 300 participants) $[14,16]$. Thus, the current study with over 3000 participants with information on multiple foot disorders may offer insights into the associations of $\mathrm{HV}$ that smaller studies cannot. Our study which included participants with $\mathrm{HV}$ and additional structural foot disorders, a typical exclusion for other HV studies [14], provides an understanding of how foot function is influenced when $\mathrm{HV}$ is coupled with additional structural foot disorders.

\section{Conclusions}

Although the pathogenesis of $\mathrm{HV}$ is complex, a better understanding of $\mathrm{HV}$ and its clinical outcomes can be achieved by evaluating the validity of theoretical, kinematic, and radiographic results through plantar pressure loading. Plantar loading can assess the functional impact of a structural deformity as seen during gait, and this study has confirmed several key results in a population- 
based sample of adult men and women with HV. Namely, a lower hallucal loading was seen in participants with $\mathrm{HV}$, with greater loading at the toes. HV and foot disorders was also associated with altered rearfoot forces, which given prior evidence suggesting forefoot complications are associated with rearfoot disorders, suggests that the rearfoot should be considered in etiology and treatment of HV and forefoot complications. In addition, lower CPEI and higher MAI values were associated with $\mathrm{HV}$, confirming results from studies that have described foot pronation and lower arch structure in feet with HV. Prospective studies are needed to elucidate of the etiology of HV and structural disorders in relation to plantar pressure loading. Moreover, longitudinal studies of $\mathrm{HV}$ and foot disorders can track plantar pressure and loading changes that develop over time.

\section{Competing interests}

The authors have no competing interests to report

\section{Authors' contributions}

AMG contributed to the analysis and interpretation of data and drafted the original manuscript. TJH contributed to the analysis and interpretation of data and drafted the original manuscript. ABD carried out the statistical analyses, contributed to the interpretation of data and the revision of the manuscript. JLR participated in the interpretation of data and the drafting and revision of the manuscript. $\mathrm{HJH}$ participated in the study conception and design and provided critical revision of the manuscript for intellectual content. VAC made substantial contributions to the drafting and revision of the manuscript. MTH conceived of the study, was responsible for the acquisition of data, contributed to the analysis and interpretation of data, and provided critical revision of the manuscript for intellectual content. All authors read and approved the final manuscript.

\section{Authors' information}

Andrew M Galica and Thomas J Hagedorn share first authorship on this manuscript.

\section{Acknowledgements}

The authors acknowledge the Framingham Foot Study research team and study participants for the contribution of their time, effort, and dedication. Funding for this project was provided by: the National Institute of Arthritis and Musculoskeletal and Skin Diseases and National Institute on Aging (AR047853). This work was derived from the Framingham Heart Study of the National Heart Lung and Blood Institute of the National Institutes of Health and Boston University School of Medicine. This work was supported by the National Heart, Lung and Blood Institute's Framingham Heart Study (Contract No. N01-HC-25195).

\section{Author details}

'Institute for Aging Research at Hebrew Senior Life, 1200 Centre Street, Boston, MA, USA. ${ }^{2}$ Beth Israel Deaconess Medical Center, Boston, MA, USA ${ }^{3}$ Harvard Medical School, Boston, MA, USA. ${ }^{4}$ Glasgow Caledonian University, Glasgow, UK. ${ }^{5}$ Hospital for Special Surgery, New York, NY, USA.

Received: 1 July 2013 Accepted: 10 October 2013

Published: 20 October 2013

\section{References}

1. Glasoe WM, Nuckley DJ, Ludewig PM: Hallux valgus and the first metatarsal arch segment: a theoretical biomechanical perspective. Phys Ther 2010, 90:110-120.

2. Nguyen US, Hillstrom HJ, Li W, Dufour AB, Kiel DP, Procter-Gray E, Gagnon MM, Hannan MT: Factors associated with hallux valgus in a populationbased study of older women and men: the MOBILIZE Boston study. Osteoarthritis Cartilage 2010, 18:41-46.
3. Roddy E, Zhang W, Doherty M: Prevalence and associations of hallux valgus in a primary care population. Arthritis Rheum 2008, 59:857-862.

4. Nix S, Smith M, Vicenzino B: Prevalence of hallux valgus in the general population: a systematic review and meta-analysis. J Foot Ankle Res 2010, 3:21.

5. Menz HB, Morris ME: Footwear characteristics and foot problems in older people. Gerontology 2005, 51:346-351.

6. Nix SE, Vicenzino BT, Collins NJ, Smith MD: Characteristics of foot structure and footwear associated with hallux valgus: a systematic review. Osteoarthritis Cartilage 2012, 20:1059-1074.

7. Schoenhaus HD, Cohen RS: Etiology of the bunion. J Foot Surg 1992, 31:25-29.

8. Kilmartin TE, Wallace WA: The significance of pes planus in juvenile hallux valgus. Foot Ankle 1992, 13:53-56.

9. Coughlin MJ: Hallux valgus. J Bone Joint Surg Am 1996, 78:932-966.

10. Schweitzer ME, Maheshwari S, Shabshin N: Hallux valgus and hallux rigidus: MRI findings. Clin Imaging 1999, 23:397-402.

11. Cancilleri F, Marinozzi A, Martinelli N, Ippolito M, Spiezia F, Ronconi P, Denaro V: Comparison of plantar pressure, clinical, and radiographic changes of the forefoot after biplanar Austin osteotomy and triplanar Boc osteotomy in patients with mild hallux valgus. Foot Ankle Int 2008, 29:817-824.

12. Schuh R, Hofstaetter SG, Adams SB Jr, Pichler F, Kristen KH, Trnka HJ: Rehabilitation after hallux valgus surgery: importance of physical therapy to restore weight bearing of the first ray during the stance phase. Phys Ther 2009, 89:934-945.

13. Wen J, Ding Q, Yu Z, Sun W, Wang Q, Wei K: Adaptive changes of foot pressure in hallux valgus patients. Gait Posture 2012, 36:344-349.

14. Martinez-Nova A, Sanchez-Rodriguez R, Perez-Soriano P, Llana-Belloch S, Leal-Muro A, Pedrera-Zamorano JD: Plantar pressures determinants in mild Hallux Valgus. Gait Posture 2010, 32:425-427.

15. Mickle KJ, Munro BJ, Lord SR, Menz HB, Steele JR: Gait, balance and plantar pressures in older people with toe deformities. Gait Posture 2011, 34:347-351.

16. Bryant $A$, Tinley $P$, Singer $K$ : Plantar pressure distribution in normal, hallux valgus and hallux limitus feet. Foot 1999, 9:115-119.

17. Menz HB, Morris ME: Clinical determinants of plantar forces and pressures during walking in older people. Gait Posture 2006, 24:229-236.

18. Dufour AB, Broe KE, Nguyen US, Gagnon DR, Hillstrom HJ, Walker AH, Kivell E, Hannan MT: Foot pain: is current or past shoewear a factor? Arthritis Rheum 2009, 61:1352-1358.

19. Hannan MT, Zimmer J, Sullivan E, Diel DP: Physical limitations and foot disorders in elders. J Am Geriatr Soc 2001, 49:S22.

20. Hannan MT, Murabito JM, Felson DT, Rivinus M, Kaplan J, Kiel DP: The epidemiology of foot disorders and foot pain in men and women: the Framingham study. Arthritis Rheum 2003, 48:S672.

21. Zammit GV, Menz HB, Munteanu SE: Reliability of the TekScan MatScan(R) system for the measurement of plantar forces and pressures during barefoot level walking in healthy adults. J Foot Ankle Res 2010, 3:11.

22. McPoil TG, Cornwall MW, Dupuis L, Cornwell M: Variability of plantar pressure data. a comparison of the two-step and midgait methods. J Am Podiatr Med Assoc 1999, 89:495-501.

23. Song J, Hillstrom HJ, Secord D, Levitt J: Foot type biomechanics. comparison of planus and rectus foot types. J Am Podiatr Med Assoc 1996, 86:16-23.

24. Chu WC, Lee SH, Chu W, Wang TJ, Lee MC: The use of arch index to characterize arch height: a digital image processing approach. IEEE Trans Biomed Eng 1995, 42:1088-1093.

25. Shiang TY, Lee SH, Lee SJ, Chu WC: Evaluating different footprint parameters as a predictor of arch height. IEEE Eng Med Biol Mag 1998, 17:62-66.

26. Nix SE, Vicenzino BT, Collins NJ, Smith MD: Gait parameters associated with hallux valgus: a systematic review. J Foot Ankle Res 2013, 6:9.

27. Van Beek C, Greisberg J: Mobility of the first ray: review article. Foot Ankle Int 2011, 32:917-922

28. Perera AM, Mason L, Stephens MM: The pathogenesis of hallux valgus. J Bone Joint Surg Am 2011, 93:1650-1661.

29. Nyska M, Liberson A, McCabe C, Linge K, Klenerman L: Plantar foot pressure distribution in patients with Hallux valgus treated by distal soft tissue procedure and proximal metatarsal osteotomy. Foot Ankle Surg 1998, 4:35-41.

30. Woodburn J, Helliwell PS: Relation between heel position and the distribution of forefoot plantar pressures and skin callosities in rheumatoid arthritis. Ann Rheum Dis 1996, 55:806-810. 
31. Keenan MA, Peabody TD, Gronley JK, Perry J: Valgus deformities of the feet and characteristics of gait in patients who have rheumatoid arthritis. J Bone Joint Surg Am 1991, 73:237-247.

32. Saragas NP, Becker PJ: Comparative radiographic analysis of parameters in feet with and without hallux valgus. Foot Ankle Int 1995, 16:139-143.

33. Blalock H: Social Statistics. New York: McGraw Hill Book Company; 1972.

34. Riskowski JL, Hagedorn TJ, Dufour AB, Hannan MT: Functional foot symmetry and its relation to lower extremity physical performance in older adults: the framingham foot study. J Biomech 2012, 45:1796-1802.

35. Hastings MK, Commean PK, Smith KE, Pilgram TK, Mueller MJ: Aligning anatomical structure from spiral X-ray computed tomography with plantar pressure data. Clin Biomech (Bristol, Avon) 2003, 18:877-882.

doi:10.1186/1757-1146-6-42

Cite this article as: Galica et al:: Hallux valgus and plantar pressure

loading: the Framingham foot study. Journal of Foot and Ankle Research 2013 6:42.

\section{Submit your next manuscript to BioMed Central and take full advantage of:}

- Convenient online submission

- Thorough peer review

- No space constraints or color figure charges

- Immediate publication on acceptance

- Inclusion in PubMed, CAS, Scopus and Google Scholar

- Research which is freely available for redistribution 